\title{
On the section conjecture and Brauer-Severi varieties
}

\author{
Giulio Bresciani ${ }^{1}$
}

Received: 25 March 2021 / Accepted: 11 June 2021 / Published online: 3 August 2021

(c) The Author(s) 2021

\begin{abstract}
J. Stix proved that a curve of positive genus over $\mathbb{Q}$ which maps to a non-trivial BrauerSeveri variety satisfies the section conjecture. We prove that, if $X$ is a curve of positive genus over a number field $k$ and the Weil restriction $R_{k / \mathbb{Q}} X$ admits a rational map to a non-trivial Brauer-Severi variety, then $X$ satisfies the section conjecture. As a consequence, if $X$ maps to a Brauer-Severi variety $P$ such that the corestriction $\operatorname{cor}_{k / \mathbb{Q}}([P]) \in \operatorname{Br}(\mathbb{Q})$ is non-trivial, then $X$ satisfies the section conjecture.
\end{abstract}

Let $X$ be a geometrically connected variety over a field $k$ with separable closure $\bar{k}$, there is a short exact sequence of étale fundamental groups

$$
0 \rightarrow \pi_{1}\left(X_{\bar{k}}\right) \rightarrow \pi_{1}(X) \rightarrow \operatorname{Gal}(\bar{k} / k) \rightarrow 0 .
$$

Grothendieck's section conjecture predicts that, if $X$ is a smooth, projective curve of genus at least 2 and $k$ is a number field, the set of rational points $X(k)$ is in natural bijection with sections of the sequence above modulo the action of $\pi_{1}\left(X_{\bar{k}}\right)$ by conjugation.

Thanks to an idea of Tamagawa [13] [12, Corollary 102] it is sufficient to prove the conjecture for curves with no rational points, and some results have been proved about such curves. The section conjecture holds for $X$ if

- the number field $k$ has a real place $k \hookrightarrow \mathbb{R}$ such that $X_{\mathbb{R}}(\mathbb{R})=\emptyset$, see [8, Corollary 3.13], or

- the class of $\underline{\mathrm{Pic}}_{X}^{1}$ in $\mathrm{H}^{1}\left(k, \underline{\mathrm{Pic}}_{X}^{0}\right)$ is not divisible, see [6, Theorem 1.2], or

- $k=\mathbb{Q}$ and $X$ maps to a non-trivial Brauer-Severi variety, see [10, Corollary 18].

The last condition, which is due to Stix, holds over any number field $k$, but with an additional hypothesis: for every prime number $p$, it is required that $X$ has bad reduction at most at one place $\mathfrak{p}$ of $k$ over $p$, see [10, Theorem 17]. We provide a different generalization based on Weil's restriction of scalars.

Recall that, given a finite separable extension $k / h$ of fields and a quasi-projective variety $X$ over $k$, the Weil restriction $R_{k / h} X$ is a quasi-projective variety over $h$ characterized by

The author is supported by the DFG Priority Program "Homotopy Theory and Algebraic Geometry" SPP 1786.

$\bowtie$ Giulio Bresciani

gbresciani@math.fu-berlin.de

1 Freie Universität Berlin, Arnimallee 7, 14195 Berlin, Germany 
a functorial bijection $\operatorname{hom}\left(S, R_{k / h} X\right) \simeq \operatorname{hom}\left(S_{k}, X\right)$ for schemes $S$ over $h$. In particular, $k$-rational points of $X$ are in natural bijection with $h$-rational points of $R_{k / h} X$.

If $X$ is a curve of genus $g$ over a number field $k$, then $\left(R_{k / \mathbb{Q}} X\right)_{\overline{\mathbb{Q}}}$ is a product of $[k: \mathbb{Q}]$ curves of genus $g$, so passing to the Weil restriction is basically a trade-off between the complexity of the base field and the complexity of the variety. We prove the following.

Theorem 1 Let $X$ be a smooth, projective, geometrically connected curve of positive genus over a number field $k$. Assume that $R_{k / \mathbb{Q}} X$ admits a rational map to a non-trivial BrauerSeveri variety. Then the section conjecture holds for $X$. Equivalently, if $\pi_{1}(X) \rightarrow \operatorname{Gal}(\bar{k} / k)$ admits a section, then the map $\operatorname{Br}(\mathbb{Q}) \rightarrow \operatorname{Br}\left(R_{k} / \mathbb{Q} X\right)$ is injective.

As a consequence, we get the following corollaries.

Corollary 2 Let $X$ be a smooth projective curve of positive genus over a number field $k$ and $P$ a Brauer-Severi variety such that the corestriction $\operatorname{cor}_{k / \mathbb{Q}}[P] \in \operatorname{Br}(\mathbb{Q})$ is non-trivial. If there exists a morphism $X \rightarrow P$, then the section conjecture holds for $X$. Equivalently, if $\pi_{1}(X) \rightarrow \operatorname{Gal}(\bar{k} / k)$ admits a section, the kernel of $\operatorname{Br}(k) \rightarrow \operatorname{Br}(X)$ is contained in the kernel of $\operatorname{cor}_{k / \mathbb{Q}}: \operatorname{Br}(k) \rightarrow \operatorname{Br}(\mathbb{Q})$.

Corollary 3 Let $k$ be a number field and P a Brauer-Severi variety over $\mathbb{Q}$ with $[k: \mathbb{Q}][P] \neq$ $0 \in \operatorname{Br}(\mathbb{Q})$. If $X$ is a smooth projective curve over $\mathbb{Q}$ of positive genus with a morphism $X \rightarrow P$, then the section conjecture holds for the base change $X_{k}$.

Our argument is analogous to Stix's one and we rely heavily on his results. Our contribution consists essentially of two things: we realized that such a generalization was possible and we overcame the lack, for higher dimensional varieties, of a sufficiently strong analogue of Lichtenbaum's theorem about the period and index of a curve over a $p$-adic field, which is an essential ingredient of Stix's proof.

We mention that it is possible to prove Corollary 2 (and thus Corollary 3) analogously to Stix' theorem over $\mathbb{Q}[10$, Corollary 18] without using Weil's restriction of scalars. The proof is basically the same plus the observation that, if $\alpha \in \operatorname{Br}(k)$ is a Brauer class over the number field $k$, the Hasse invariant of $\operatorname{cor}_{k / \mathbb{Q}}(\alpha)$ at $p$ is the sum of the Hasse invariants of $\alpha$ at places over $p$.

\section{Weil restriction and the section conjecture}

The behaviour of the étale fundamental group and the section conjecture with respect to the Weil restriction of scalars has been studied by J. Stix in [11]. Let $k / h$ be a finite, separable extension of fields and $X$ is a geometrically connected variety over $k$. Assume either that $X$ is proper or that $k$ has characteristic 0 . Stix describes explicitly the étale fundamental group of $R_{k / h} X$ in terms of the one of $X$, and uses this description to show that the section conjecture holds for $X$ if and only if it holds for $R_{k / h} X$. We give here an alternative treatment based on étale fundamental gerbes.

Recall that A. Vistoli and N. Borne have introduced the étale fundamental gerbe $X \rightarrow$ $\Pi_{X / k}$ of a geometrically connected scheme, see [4, Section 9] and [3, Appendix]. The set of Galois sections of the étale fundamental group is in natural bijection with the isomorphism classes of $\Pi_{X / k}(k)$. We show that the étale fundamental gerbe and the Weil restriction commute.

Proposition 4 Let $k / h$ be a finite separable extension of fields, and X a geometrically connected quasi-projective variety over $k$. Assume either that $X$ is proper or that char $k=0$. 
Then $R_{k / h} X$ is geometrically connected and the natural morphism $R_{k / h} X \rightarrow R_{k / h} \Pi_{X / k}$ induces a natural isomorphism

$$
\Pi_{R_{k / h} X / h} \stackrel{\sim}{\rightarrow} R_{k / h} \Pi_{X / k}
$$

Proof Let $\bar{h} / h$ be a separable closure. We have natural isomorphisms $\left(R_{k / h} X\right)_{\bar{h}}=\prod \sigma^{*} X$ and $\left(R_{k / h} \Pi_{X / k}\right)_{\bar{h}} \simeq \prod \sigma^{*} \Pi_{X / k}=\prod \Pi_{\sigma^{*} X / k}$ where the product runs over the $h$-linear embeddings $\sigma: k \subset \bar{h}$, see [14, Theorem 1.3.2] (Weil's original work deals only with varieties, but his proof easily generalizes to any fibered category). In particular $R_{k / h} X$ is geometrically connected and $R_{k / h} \Pi_{X / k}$ is a pro-finite étale gerbe, thus the morphism $R_{k / h} X \rightarrow R_{k / h} \Pi_{X / k}$ induces a natural morphism

$$
\phi: \Pi_{R_{k / h} X / h} \rightarrow R_{k / h} \Pi_{X / k}
$$

The base change of $\phi$ to $\bar{h}$ is an isomorphism since both terms are naturally isomorphic to $\prod_{\sigma} \Pi_{\sigma^{*} X / k}$, thus $\phi$ is an isomorphism too.

Corollary 5 [11, Theorem 2, Theorem 3] The set of isomorphism classes of Galois sections of $\pi_{1}(X)$ over $k$ is in natural bijection with the one of $\pi_{1}\left(R_{k / h} X\right)$ over $h$.

Corollary 6 [11, Theorem 4] Let $X$ be a smooth, projective curve of genus $g \geq 2$ over a number field $k$. The section conjecture holds for $X$ if and only if it holds for $R_{k / \mathbb{Q}} X$.

If $s \in \Pi_{X / k}(k)$ is a Galois section, denote by $s^{\mathbb{Q}} \in \Pi_{R_{k} / \mathbb{Q}} X / \mathbb{Q}(\mathbb{Q})=R_{k / \mathbb{Q}} \Pi_{X / \mathbb{Q}}(\mathbb{Q})$ the induced section. Recall that an étale neighbourhood of $s$ is a finite étale cover $Y \rightarrow X$ such that $s$ lifts to $\Pi_{Y / k}(k)$.

Corollary 7 Let $X$ be a smooth, projective curve of positive genus over a number field $k$ and $s \in \Pi_{X / k}(k)$ a Galois section. If $Y \rightarrow X$ is an étale neighbourhood of $s$, then $R_{k / \mathbb{Q}} Y \rightarrow$ $R_{k / \mathbb{Q}} X$ is an étale neighbourhood of $s \mathbb{Q}$. The étale neighbourhoods of this form are cofinal in the system of all étale neighbourhoods of $s \mathbb{Q}$.

\section{Morphisms to Brauer-Severi varieties}

If $X$ is a scheme over $k$, denote by $\operatorname{Br}(X / k)$ the kernel of $\operatorname{Br}(k) \rightarrow \operatorname{Br}(X)$. If $X$ is a regular variety, the restriction map $\operatorname{Br}(X) \rightarrow \operatorname{Br}(k(X))$ is injective [7, Corollary IV.2.6] and thus $\operatorname{Br}(X / k)=\operatorname{Br}(k(X) / k)$. In particular, a Brauer class $[P] \in \operatorname{Br}(k)$ of a Brauer-Severi variety $P$ is in $\operatorname{Br}(X / k)$ if and only if there exists a rational map $X \rightarrow P$.

If $X$ is a smooth, projective variety, the Leray spectral sequence in étale cohomology for the map $X \rightarrow$ Spec $k$ gives a short exact sequence

$$
0 \rightarrow \operatorname{Pic}(X) \rightarrow \underline{\operatorname{Pic}}_{X}(k) \rightarrow \operatorname{Br}(X / k) \rightarrow 0
$$

where $\underline{\operatorname{Pic}}_{X}$ is the Picard scheme of $X$ and $\operatorname{Pic}(X)=\mathrm{H}^{1}\left(X, \mathbb{G}_{m}\right)$ is the Picard group. Let us call $\beta$ the homomorphism $\underline{\operatorname{Pic}}_{X}(k) \rightarrow \operatorname{Br}(X / k)$.

Lemma 8 Let $X$ be a smooth, projective variety over a field $k$ of characteristic $0, s \in \Pi_{X / k}(k)$ a section, $b \in \operatorname{Br}(X / k)$ a Brauer class split by $X$. Assume that the second étale homotopy group of $X_{\bar{k}}$ is trivial. For every positive integer $n$, there exists an étale neighbourhood $f: Y \rightarrow X$ of s such that $f^{*} b \in n \operatorname{Br}(Y / k)$. 
Proof Let $L \in \underline{\operatorname{Pic}}_{X}(k)$ be such that $\beta(L)=b \in \operatorname{Br}(X / k)$, and $L_{\bar{k}} \in \operatorname{Pic}\left(X_{\bar{k}}\right)$ the associated line bundle over $X_{\bar{k}}$. We have an exact sequence

$$
\mathrm{H}^{1}\left(X_{\bar{k}}, \mu_{n}\right) \rightarrow \operatorname{Pic}\left(X_{\bar{k}}\right) \stackrel{\cdot n}{\rightarrow} \operatorname{Pic}\left(X_{\bar{k}}\right) \stackrel{\delta}{\rightarrow} \mathrm{H}^{2}\left(X_{\bar{k}}, \mu_{n}\right) . s
$$

Let Ét $\left(X_{\bar{k}}\right)$ be the étale homotopy type of $X_{\bar{k}}$ and $\operatorname{cosk}_{3}\left(\operatorname{E} t\left(X_{\bar{k}}\right)\right)$ its third coskeleton, since $\pi_{2}^{\mathrm{Ket}}\left(X_{\bar{k}}\right)$ is trivial we have $\operatorname{cosk}_{3}\left(\operatorname{Ét}\left(X_{\bar{k}}\right)\right)=K\left(\pi_{1}^{\mathrm{Ket}}\left(X_{\bar{k}}\right), 1\right)$. Therefore, we have

$$
\mathrm{H}^{2}\left(X_{\bar{k}}, \mu_{n}\right)=\mathrm{H}^{2}\left(\operatorname{Ét}\left(X_{\bar{k}}\right), \mu_{n}\right)=\mathrm{H}^{2}\left(\operatorname{cosk}_{3}\left(\operatorname{Ét}\left(X_{\bar{k}}\right)\right), \mu_{n}\right)=\mathrm{H}^{2}\left(\pi_{1}^{\operatorname{Ket}}\left(X_{\bar{k}}\right), \mu_{n}\right),
$$

see [1, Corollary 9.3] for the first equality. Since the base change to $\bar{k}$ of the étale neighbourhoods of $s$ are cofinal in all finite étale covers of $X_{\bar{k}}$, there exists an étale neighbourhood $g: X^{\prime} \rightarrow X$ of $s$ such that $g_{\bar{k}}^{*} \delta\left(L_{\bar{k}}\right)=0$ and thus $g_{\bar{k}}^{*} L_{\bar{k}} \in \operatorname{Pic}\left(X_{\bar{k}}^{\prime}\right)$ is divisible by $n$.

Choose $M \in \operatorname{Pic}\left(X_{\bar{k}}^{\prime}\right)$ such that $M^{n}=g_{\bar{k}}^{*} L_{\bar{k}}$. Since the Picard scheme of $X^{\prime}$ is locally of finite type, the residue field of Spec $\bar{k} \stackrel{M}{\longrightarrow} \underline{\mathrm{Pic}}_{X^{\prime}}$ is finite over $k$ and thus the Galois orbit of $M$ is finite. If $\sigma \in \mathrm{Gal}(\bar{k} / k)$ is an element, since $g_{\bar{k}}^{*} L_{\bar{k}}$ is Galois invariant then $M \otimes \sigma M^{-1}$ is $n$-torsion, and thus it comes from $\mathrm{H}^{1}\left(X_{\bar{k}}^{\prime}, \mu_{n}\right)=\operatorname{hom}\left(\pi_{1}^{\mathrm{Ket}}\left(X_{\bar{k}}^{\prime}\right), \mu_{n}\right)$. It follows that there exists an étale neighbourhood $h: Y \rightarrow X^{\prime}$ of $s$ such that $h_{\vec{k}}^{*} M \in \operatorname{Pic}\left(Y_{\bar{k}}\right)$ is Galois-invariant and thus descends to an element $N \in \underline{\operatorname{Pic}}_{Y}(k)$.

Let $f: Y \rightarrow X$ be the composition, we have $N^{n}=f^{*} L$ and hence $n \beta(N)=\beta\left(f^{*} L\right)=$ $f^{*} b$.

Recall that a group $G$ is good in the sense of Serre if $\mathrm{H}^{i}(\hat{G}, M) \rightarrow \mathrm{H}^{i}(G, M)$ is an isomorphism for every finite $G$-module $M$, see [9, I.2.6]. Fundamental groups of complex curves are good [5, Proposition 3.6].

Lemma 9 Let $X$ be a variety over $\mathbb{C}$. Assume that $\pi_{2}^{\text {top }}\left(X^{\mathrm{an}}\right)$ is trivial and that $\pi_{1}^{\text {top }}\left(X^{\mathrm{an}}\right)$ is good in the sense of Serre. Then $\pi_{2}^{\mathrm{Ket}}(X)$ is trivial.

Proof Write $\pi_{i}=\pi_{i}^{\mathrm{Ket}}(X)$. The hypothesis implies that the natural homomorphism $\mathrm{H}^{2}\left(\pi_{1}, M\right) \rightarrow \mathrm{H}^{2}(X, M)$ is bijective for every finite $\pi_{1}$-module $M$.

Assume by contradiction that $\pi_{2}$ is not trivial, then there exists a finite homotopy type $F$ with $\pi_{n}(F)=0$ for $n \geq 3$ and a map Ét $(X) \rightarrow F$ such that $\pi_{2} \rightarrow \pi_{2}(F)$ is non-trivial. Up to passing to finite étale coverings of $X$ and $F$, we may assume that $\pi_{1}(F)$ is trivial and hence $F=K(M, 2)$ for some finite abelian group $M$ (the fundamental group of the covering of $X$ is still good thanks to [5, Lemma 3.2]).

We thus have a map $X \rightarrow K(M, 2)$ inducing a non-trivial homomorphism $\pi_{2} \rightarrow M$. This defines a cohomology class $\alpha \in \mathrm{H}^{2}(X, M)$ not in the image of $\mathrm{H}^{2}\left(\pi_{1}, M\right) \rightarrow \mathrm{H}^{2}(X, M)$, and this is absurd.

Lemma 10 Let $k / h$ be a finite separable extension and $P / k$ a Brauer-Severi variety. There exists a Brauer-Severi variety $Q / h$ with $[Q]=\operatorname{cor}_{k / h}([P]) \in \operatorname{Br}(h)$ and a closed embedding $R_{k / h} P \hookrightarrow Q$.

Proof Let $\bar{h} / h$ be a separable closure, then

$$
R_{k / h} P=\left(\prod_{\sigma} \sigma^{*} P\right) / \operatorname{Gal}(\bar{h} / h) s
$$

where the product runs over $h$-linear embeddings $\sigma: k \rightarrow \bar{h}$. The Galois group $\operatorname{Gal}(\bar{h} / h)$ permutes the factors and the stabilizer $\operatorname{Gal}(\bar{h} / \sigma k)$ of $\sigma^{*} P$ acts on it. Note that, even though $\sigma^{*} P$ is a projective space over $\bar{h}$, the action of $\operatorname{Gal}(\bar{h} / \sigma k)$ is non-standard. 
The external tensor product $\bigotimes_{\sigma} \mathcal{O}(1) \in \operatorname{Pic}\left(\prod \sigma^{*} P\right)$ is naturally $\operatorname{Gal}(\bar{h} / h)$-equivariant and thus the Segre embedding

$$
S: \prod_{\sigma} \sigma^{*} P \hookrightarrow \mathbb{P}\left(\mathrm{H}^{0}\left(\bigotimes_{\sigma} \mathcal{O}(1)\right)\right)
$$

is naturally $\operatorname{Gal}(\bar{h} / h)$-equivariant. The quotient $Q=\mathbb{P}\left(\mathrm{H}^{0}\left(\bigotimes_{\sigma} \mathcal{O}(1)\right)\right) / \mathrm{Gal}(\bar{h} / h)$ is a BrauerSeveri variety over $h$.

Using the fact that summation in the Brauer group can be computed using the Segre embedding of Brauer-Severi varieties $[2, \S 4]$ and the fact that the corestriction homomorphism is the derived augmentation homomorphism, it is easy to show that that $[Q]=\operatorname{cor}_{k / h}([P])$. Moreover, the Segre embedding $S$ descends to a closed embedding $R_{k / h} P \hookrightarrow Q$ since it is $\operatorname{Gal}(\bar{h} / h)$-equivariant.

\section{Proof of the main theorem}

Let us now prove Theorem 1. Let $X$ be a smooth projective curve over a number field $k$ such that $R_{k / \mathbb{Q}} X$ admits a rational map to a non-trivial Brauer-Severi variety, we want to show that $\Pi_{X / k}(k)$ is empty. Assume by contradiction that there exists a section $s \in \Pi_{X / k}(k)$ and let $b \in \operatorname{Br}\left(R_{k / \mathbb{Q}} X / \mathbb{Q}\right)$ be a non-trivial Brauer class. Let $s^{\mathbb{Q}} \in \Pi_{R_{k / \mathbb{Q}}} X / \mathbb{Q}(\mathbb{Q})$ be the associated section.

Since $\left(R_{k / \mathbb{Q}} X\right)_{\bar{k}}$ is a product of curves and the fundamental group of a curve over $\bar{k}$ is good in the sense of Serre [5, Proposition 3.6], then Lemma 9 implies that $\pi_{2}^{\operatorname{Ket}}\left(\left(R_{k / \mathbb{Q}} X\right)_{\bar{k}}\right)$ is trivial and we may thus apply Lemma 8 to $R_{k / \mathbb{Q}} X$ and $s{ }^{\mathbb{Q}}$. If we apply Lemma 8 together with Corollary 7, for every $N>0$ we may find an étale neighbourhood $X_{N} \rightarrow X$ of $s$ and a Brauer class $b_{N} \in \operatorname{Br}\left(R_{k / \mathbb{Q}} X_{N} / \mathbb{Q}\right)$ such that $N b_{N}=b \in \operatorname{Br}(\mathbb{Q})$ is non-trivial.

Let $l / k / \mathbb{Q}$ be a Galois closure. Up to replacing $X$ with $X_{2[l: \mathbb{Q}]}$ and $b$ with $b_{2[l: \mathbb{Q}]}$, we may assume that $2[l: \mathbb{Q}] b \in \operatorname{Br}(\mathbb{Q})$ is non-trivial.

Fix $p$ a prime number, let us show that the order of the Brauer class $2[l: \mathbb{Q}] b_{\mathbb{Q}_{p}}$ is a power of $p$. Let $L$ be the completion of $l$ at some place over $p$, we have that $L / \mathbb{Q}_{p}$ is a Galois extension such that $\left[L: \mathbb{Q}_{p}\right]$ divides $[l: \mathbb{Q}]$, it is enough to show that the order of $\left[L: \mathbb{Q}_{p}\right] b_{\mathbb{Q}_{p}}$ is a power of $p$. Let $\Sigma$ be the set of embeddings $k \rightarrow L$, we have

$$
\left(R_{k / \mathbb{Q}} X\right)_{L}=\prod_{\sigma \in \Sigma} \sigma^{*} X .
$$

The section $s \in \Pi_{X / k}(k)$ induces a section $\sigma^{*} s \in \Pi_{\sigma^{*} X / L}(L)$ for every embedding $\sigma: k \rightarrow$ $L$. By [12, Theorem 15], this implies that the index of $\sigma^{*} X$ is a power of $p$ for every $\sigma$. Let $D_{\sigma} \in Z_{0}\left(X_{\sigma}\right)$ be a 0 -cycle whose degree is a power of $p$, then $\otimes_{\sigma} D_{\sigma}$ is a 0 -cycle on $\prod_{\sigma} \sigma^{*} X$ whose degree is a power of $p$. It follows that the index of $\prod_{\sigma} \sigma^{*} X$ is a power of $p$, too.

Since $\mathbb{Q}\left(R_{k / \mathbb{Q}} X\right)$ splits $b$, there exists a Brauer-Severi variety $P$ with $[P]=b$ and a smooth projective variety $Y / \mathbb{Q}$ birational to $R_{k} \mathbb{Q} X$ with a morphism $Y \rightarrow P$. Since the index is a birational invariant, the index of $Y_{L}$ is a power of $p$, it follows that the index of $b_{L}=\left[P_{L}\right]$ is a power of $p$. This implies that the order (i.e. the period) of $b_{L} \in \operatorname{Br}(L)$ is a power of $p$, and finally the same holds for $\operatorname{cor}_{L / \mathbb{Q}_{p}} b_{L}=\left[L: \mathbb{Q}_{p}\right] b_{\mathbb{Q}_{p}}$.

We thus have that the order of $2[l: \mathbb{Q}] b_{\mathbb{Q}_{p}}$ is $p$-primary for every $p$, and clearly $2[l$ : $\mathbb{Q}] b_{\mathbb{R}}=0 \in \operatorname{Br}(\mathbb{R})=\mathbb{Z} / 2 \mathbb{Z}$.

The rest of the argument is analogous to Stix's one. Let $\alpha_{p} \in \mathbb{Q} / \mathbb{Z}$ be the Hasse invariant of $2[l: \mathbb{Q}] b_{\mathbb{Q}_{p}}$, by the Brauer-Hasse-Noether theorem we have $\sum_{p} \alpha_{p}=0 \in \mathbb{Q} / \mathbb{Z}$. Since 
$\alpha_{p}$ is $p$-primary for every $p$, it follows that $\alpha_{p}=0$ for every $p$ and thus $2[l: \mathbb{Q}] b \in \operatorname{Br}(\mathbb{Q})$ is trivial, which is absurd. This concludes the proof of Theorem 1.

\section{Corollaries}

Theorem 1 implies Corollary 2 using Lemma 10. Since the composition $\operatorname{cor}_{k / \mathbb{Q}} \circ \operatorname{res}_{k / \mathbb{Q}}$ : $\operatorname{Br}(\mathbb{Q}) \rightarrow \operatorname{Br}(\mathbb{Q})$ is multiplication by $[k: \mathbb{Q}]$, Corollary 2 implies Corollary 3.

Acknowledgements Lemma 8 was found during joint work with A. Vistoli. I would like to thank an anonymous referee for many useful remarks.

Funding Open Access funding enabled and organized by Projekt DEAL.

Open Access This article is licensed under a Creative Commons Attribution 4.0 International License, which permits use, sharing, adaptation, distribution and reproduction in any medium or format, as long as you give appropriate credit to the original author(s) and the source, provide a link to the Creative Commons licence, and indicate if changes were made. The images or other third party material in this article are included in the article's Creative Commons licence, unless indicated otherwise in a credit line to the material. If material is not included in the article's Creative Commons licence and your intended use is not permitted by statutory regulation or exceeds the permitted use, you will need to obtain permission directly from the copyright holder. To view a copy of this licence, visit http://creativecommons.org/licenses/by/4.0/.

\section{References}

1. Artin, M., Mazur, B.: Etale Homotopy. LectureNotes in Mathematics 100. Springer-Verlag (1969)

2. Artin, M.: Brauer-Severi varieties. In: Brauer groups in ring theory and algebraic geometry (Wilrijk, 1981). LectureNotes in Math, vol. 917, pp. 194-210. Springer, Berlin-NewYork (1982)

3. Borne, N., Vistoli,A.: The Nori fundamental gerbe of a fibered category. In: Journal of Algebraic Geometry 24 (2 Apr. 2015), pp. 311-353

4. Bresciani, G.: Some implications between Grothendieck's anabelian conjectures. In: Algebr. Geom. 8.2 (2021), pp. 231-267. ISSN: 2313-1691. https://doi.org/10.14231/ag-2021-005

5. Grunewald, F., Jaikin-Zapirain, A., Zalesskii, P.A.: Cohomological goodness and the profinite completion of Bianchi groups. Duke Math. J. 144(1), 53-72 (2008)

6. Harari,D., Szamuely, T.: Galois sections for abelianized fundamental groups. In: Math. Ann. 344.4 (2009). With an appendix by E. V. Flynn, pp. 779-800. ISSN: 0025-5831. https://doi.org/10.1007/s00208-0080327-z.

7. Milne, J. S.: Étale cohomology. Princeton Mathematical Series, vol. 33, pp. xiii+323. PrincetonUniversity Press, Princeton, N.J. (1980). ISBN: 0-691-08238-3

8. Mochizuki, S.: Topics surrounding the anabelian geometry of hyperbolic curves. In: Galois groups and fundamental groups. Vol. 41. Math. Sci. Res. Inst. Publ. Cambridge Univ. Press, Cambridge, 2003, pp. 119-165

9. Serre, J.-P.: Cohomologie galoisienne. Fifth. Lecture Notes in Mathematics, vol. 5, pp. x+181. SpringerVerlag, Berlin (1994). ISBN: 3-540-58002-6. https://doi.org/10.1007/BFb0108758

10. Stix, J.: Rational points and arithmetic of fundamental groups. Lecture Notes in Mathematics 2054. Springer, 2013

11. Stix, J.: On the period-index problem in light of the section conjecture. Am. J. Math. 132(1), 157-180 (2010)

12. Stix, J.: Trading degree for dimension in the section conjecture: The non-abelian Shapiro lemma. Math. J. Okayama Univ. 52, 29-43 (2010)

13. Tamagawa, A.: The Grothendieck conjecture for affine curves. CompositioMathematica 109(2), 135-194 (1997)

14. Weil, A.: Adeles and algebraic groups. Vol. 23. Progress in Mathematics. With appendices by M. Demazure and Takashi Ono. Birkhäuser, Boston, Mass., 1982, pp. iii+126. ISBN: 3-7643-3092-9

Publisher's Note Springer Nature remains neutral with regard to jurisdictional claims in published maps and institutional affiliations. 